\title{
Fish-scale bio-inspired multifunctional ZnO nanostructures
}

\author{
Ziqi Sun ${ }^{1,2}$, Ting Liao', Wenxian $\mathrm{Li}^{3}$, Yuhai Dou${ }^{1}$, Kesong Liu ${ }^{4}$, Lei Jiang ${ }^{4,5}$, Sang-Woo Kim ${ }^{6}$, Jung Ho Kim ${ }^{1}$ and \\ Shi Xue Dou ${ }^{1}$
}

Scales provide optical disguise, low water drag and mechanical protection to fish, enabling them to survive catastrophic environmental disasters, predators and microorganisms. The unique structures and stacking sequences of fish scales inspired the fabrication of artificial nanostructures with salient optical, interfacial and mechanical properties. Herein, we describe fish-scale bio-inspired multifunctional $\mathrm{ZnO}$ nanostructures that have similar morphology and structure to the cycloid scales of the Asian Arowana. These nanostructured coatings feature tunable light refraction and reflection, modulated surface wettability and damage-tolerant mechanical properties. The salient properties of these multifunctional nanostructures are promising for applications in (i) optical coatings, sensing or lens arrays for use in reflective displays, packing, advertising and solar energy harvesting; (ii) self-cleaning surfaces, including anti-smudge, anti-fouling and anti-fogging, and self-sterilizing surfaces; and (iii) mechanical/chemical barrier coatings. This study provides a low-cost and large-scale production method for the facile fabrication of these bio-inspired nanostructures and provides new insights for the development of novel functional materials for use in 'smart' structures and applications.

NPG Asia Materials (2015) 7, e232; doi:10.1038/am.2015.133; published online 18 December 2015

\section{INTRODUCTION}

In the past decade, techniques have been rapidly developed to produce 'smart' multifunctional nanomaterials by applying lessons learned from nature, materials termed 'bio-inspired nanostructures. ${ }^{1,2}$ This approach has naturally led to the development of bio-inspired multifunctional nanomaterials for use in various applications. For example, several researchers have described lotus-leaf-inspired self-cleaning surfaces, plant- and insect-inspired anisotropic superhydrophobic surfaces, fly-eye-inspired anti-fogging coatings, insect-inspired antireflection coatings, rose-petal- and gecko-footinspired highly adhesive surfaces, cactus-inspired fog-collecting surfaces and butterfly-wing-inspired optical materials. ${ }^{1-9}$ These materials are based on the fact that biological species have optimal structures that have been honed through millions of years of evolution and that exhibit amazing characteristics and swift stimulus-responsive capabilities, which provide inspiration to researchers for the design of multifunctional materials.

Fish have existed for $\sim 500 \mathrm{Myr}$ and have survived several catastrophic mass extinction events during global environmental disasters. ${ }^{10}$ Most fish have hard scales on their skin for protection. It has been suggested that the dermal armor of ancient fish served as a protection from predators and mechanical damage, and maximized survivability. ${ }^{11}$ Generally, fish scales serve three major functions: (i) providing mechanical protection from external mechanical disturbances and from invasion by bacteria and other microorganisms; (ii) providing low drag mobility in water to improve maneuverability and speed; and (iii) providing camouflage protection via light reflection and refraction to escape predators. ${ }^{10}$ The unique structures and functionalities of fish scales have attracted great interest, and some progress has been made in understanding their crystalline growth, morphologies, small length scale effects and fundamental properties. One of the most famous cases arising from this greater knowledge is a shark-skin-inspired swimsuit that has boosted swimming speed through its ability to reduce drag. ${ }^{12}$ An excellent bio-inspired surface that features underwater superoleophobicity has been fabricated by replicating shark pacoid and cycloid scales. ${ }^{13}$ Ortiz et al. have studied the mechanical properties of individual dermal armor plates and scales, and have further proposed design principles for bio-inspired

\footnotetext{
${ }^{1}$ Institute for Superconducting and Electronic Materials, University of Wollongong, Innovation Campus, North Wollongong, NSW, Australia; ${ }^{2}$ School of Chemistry, Physics and Mechanical Engineering, Queensland University of Technology, Brisbane, QLD, Australia; ${ }^{3}$ School of Materials Science and Engineering, Shanghai University, Shanghai, China; ${ }^{4}$ Key Laboratory of Bio-inspired Smart Interfacial Science and Technology of the Ministry of Education, School of Chemistry and the Environment, Beihang University, Beijing, China; ${ }^{5}$ Beijing National Laboratory for Molecular Sciences (BNLMS), Key Laboratory of Organic Solids, Institute of Chemistry, Chinese Academy of Sciences, Beijing, China and ${ }^{6}$ School of Advanced Materials Science and Engineering, SKKU Advanced Institute of NanoTechnology, Sungkunkwan University, Gyeonggi-do, Korea

Correspondence: Professor JH Kim, Institute for Superconducting and Electronic Materials, University of Wollongong, Innovation Campus, North Wollongong, 2500 NSW, Australia.

E-mail: jhk@uow.edu.au

or Professor L Jiang, Beijing National Laboratory for Molecular Sciences (BNLMS), Key Laboratory of Organic Solids, Institute of Chemistry, Chinese Academy of Sciences, Beijing 100190, China.

E-mail: jianglei@iccas.as.cn
}

Received 19 June 2015; revised 2 September 2015; accepted 28 September 2015 
human body armor. ${ }^{11}$ Even though several reports have described studies of fish scales and their replica surfaces, no relevant reports can be found describing the facile synthesis of fish-scale bio-inspired inorganic nanostructures that can mimic their multiscale structures and multiple functions. The development of fish-scale bio-inspired inorganic nanostructures in this study will consequently greatly extend the use of bio-inspired materials to applications including micromechanical devices, heavy-duty machines (as protective coatings against mechanical damage and chemical corrosion), optical devices (as optical elements), photovoltaics and low-drag or low-friction surfaces in gaseous, liquid and solid media.
Herein, we describe the design of fish-scale bio-inspired multifunctional $\mathrm{ZnO}$ nanostructures that have similar surface structures to the cycloid scales of the Asian Arowana. The Asian Arowana (Scleropages), a member of Osteoglossidae and of the earliest fish to appear ( $340 \mathrm{Myr}$ ago during the Carboniferous period) continues to exist today. ${ }^{14}$ Figure 1a illustrates an Asian Arowana fish, which are usually $\sim 60 \mathrm{~cm}$ in length and covered with large cycloid scales, each of which is generally longer than $2 \mathrm{~cm}$. An enlarged image of the scales is shown in Figure 1b. In this study, bio-inspired $\mathrm{ZnO}$ nanostructures in the form of isolated microspheres (Figures 1c and d) and large-scale coatings (Figure 2), both of which have surfaces with a similar stacking

a

b

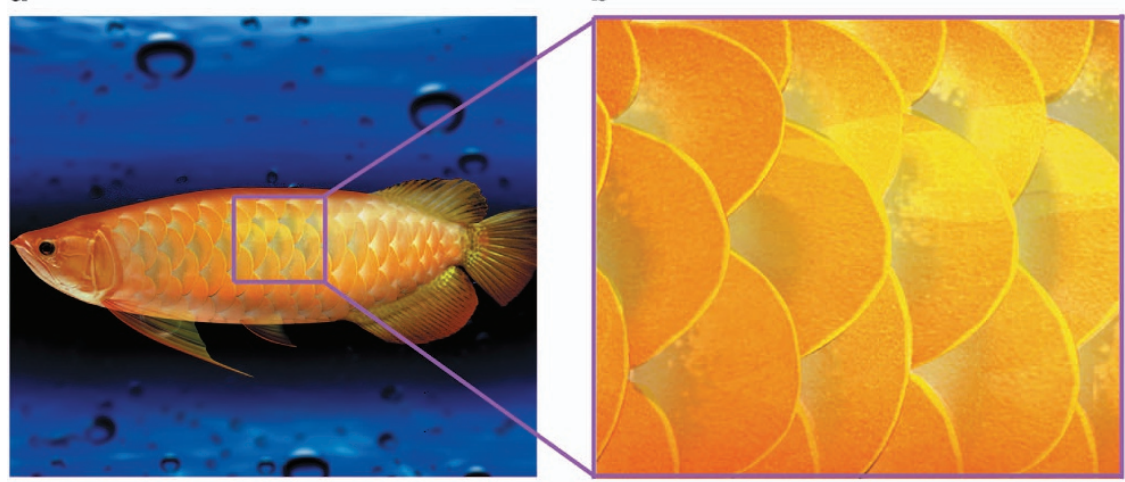

C

d

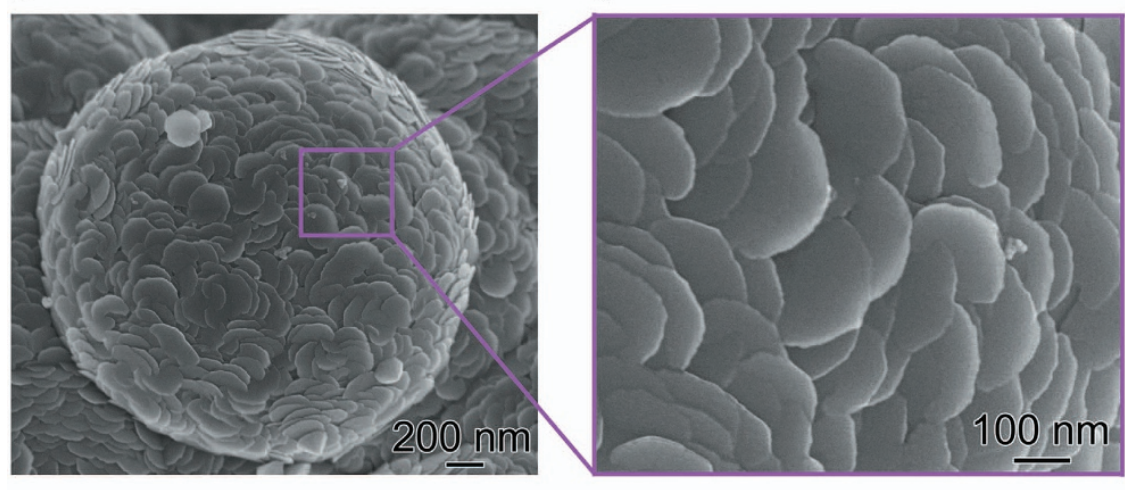

e

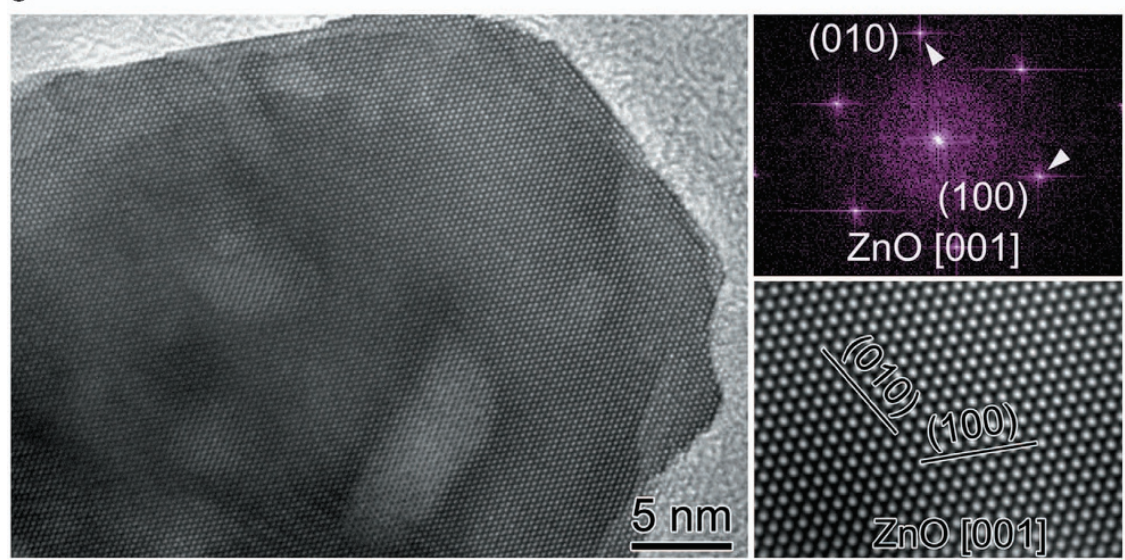

Figure 1 Microstructure of natural Asia Arowana fish-scale and bio-inspired nanostructures: (a) an optical image of an Asian Arowana fish, (b) an enlarged optical image of Asia Arowana fish scales; (c) a scanning electron microscope (SEM) image of ZnO microspheres covered with fish-scale bio-inspired nanostructures, (d) a high-magnification SEM image of the fish-scale bio-inspired nanostructures showing a similar stacking sequence to that of natural fish scales and (e) a high-resolution transmission electron microscope image of the fish-scale-like $\mathrm{ZnO}$ nanostructure (left), the corresponding fast Fourier transform (FFT) pattern (right top) and the enlarged lattice image (right bottom). 
a

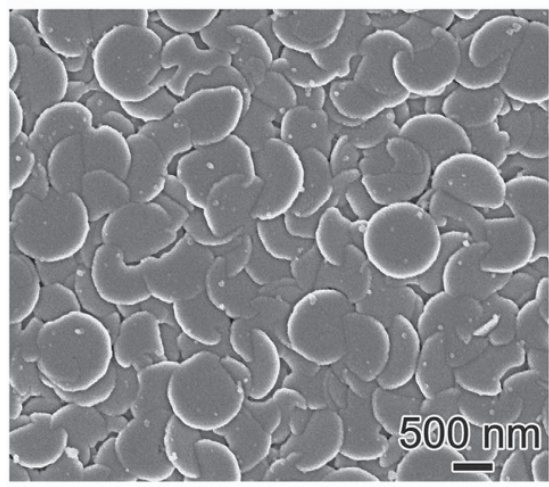

d

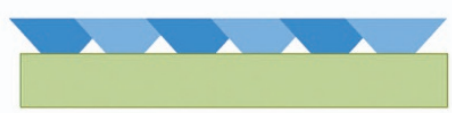

low seed density

$130^{\circ} \mathrm{C}$ b
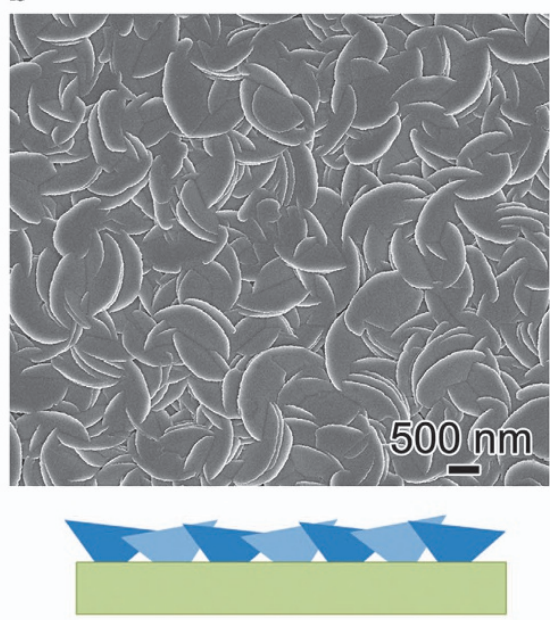

low seed density

$150^{\circ} \mathrm{C}$ c
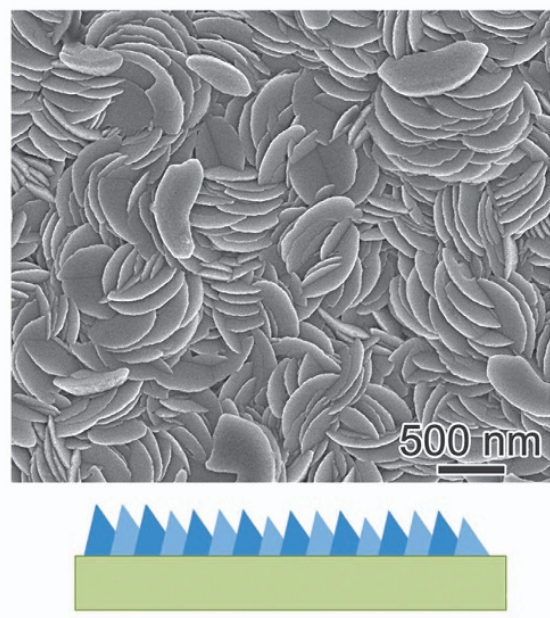

high seed density

$150^{\circ} \mathrm{C}$

Figure 2 Fish-scale bio-inspired nanostructured coatings on FTO glass substrates. (a) A scanning electron microscope (SEM) image of the bio-inspired coating with parallel scale-like nanostructures, (b) a SEM image of the bio-inspired coating with parallel scale-like nanostructures mixed with slightly tilted scales and (c) a SEM image of the bio-inspired coating with tilted scale-like nanostructures; (d) a schematic drawing showing the fish-scale-like nanostructured coatings with different scale orientations grown under different synthesis conditions.

sequence to that of the fish scales, were synthesized via a facile hydrothermal process. $\mathrm{ZnO}$ is a typical inorganic metal oxide that is easily fabricated into a variety of morphologies to meet different functional requirements. ${ }^{15-19}$ Both the structure and the surface composition of the fish scales are reportedly crucial for providing low drag mobility to improve the maneuverability and speed of fish in water. ${ }^{20}$ Therefore, appropriate surface modifications were also carried out on the bio-inspired nanostructured coatings. To explore the multifunctional applications of these unique bio-inspired nanostructures, their optical properties, surface wettability modulation (to superhydrophilicity or superhydrophobicity) and mechanical properties were studied. For the bio-inspired materials, the nanoscale properties are expected to be more interesting than those on the millimeter scale. On the basis of this consideration, we controlled the bio-inspired $\mathrm{ZnO}$ crystals at a nanometer scale in this study.

\section{EXPERIMENTAL PROCEDURES}

\section{Synthesis}

To prepare reaction solutions for the synthesis of the bio-inspired nanostructures, polyethylene oxide-polypropylene oxide-polyethylene oxide $\left(\mathrm{PEO}_{20}-\mathrm{PPO}_{70}-\mathrm{PEO}_{20}\right.$, Pluronic P123) surfactant and precisely controlled $\mathrm{H}_{2} \mathrm{O}$ and ethylene glycol (EG) co-surfactant were added to $3 \mathrm{ml}$ ethanol to form a surfactant solution. Then, $\mathrm{ZnAc}_{2} \cdot 2 \mathrm{H}_{2} \mathrm{O}$ was added to the surfactant solution, which was then stirred for $\sim 10 \mathrm{~min}$. Next, hexamethylenetetramine was added, and stirring was maintained for another $10 \mathrm{~min}$ to allow dissolution of all the crystals. After further stirring for $\sim 3 \mathrm{~h}$, a transparent solution was obtained. The transparent precursor solution was statically aged for 4 days. Then, the well-aged reaction solutions were transferred into an autoclave and heated at $100-150^{\circ} \mathrm{C}$ for $5-15 \mathrm{~h}$.

The fish-scale bio-inspired isolated microspheres were synthesized from the aged solution, which contained $0.8 \mathrm{~g} \mathrm{ZnAc} \cdot 2 \mathrm{H}_{2} \mathrm{O}, 0.5 \mathrm{~g}$ hexamethylenetetramine, $0.42 \mathrm{~g} \mathrm{H}_{2} \mathrm{O}$ and $12 \mathrm{ml} \mathrm{EG}$, and was then solvothermally heated at $110^{\circ} \mathrm{C}$ for $15 \mathrm{~h}$. To grow the bio-inspired nanostructured coatings, clean glass substrates with different $\mathrm{ZnO}$ nanoseed densities were placed into the aged reaction solutions and then solvothermally treated at $90-150^{\circ} \mathrm{C}$. Before the growth of the nanostructured coatings, a thin layer of $\mathrm{ZnO}$ seeds was carefully pre-deposited on the substrates. The seed solution used for low-density seed deposition was $20 \mathrm{ml}$ of an ethanol solution containing $0.02 \mathrm{~g} \mathrm{ZnAc} \cdot 2 \mathrm{H}_{2} \mathrm{O}$. Seed deposition was carried out by spin coating the well-stirred seed solution onto the substrates at 2,000 r.p.m. for $30 \mathrm{~s}$; this procedure was repeated 1-5 times at intervals of $15 \mathrm{~min}$, depending on the case. After seed coating, the substrates were heated at $400{ }^{\circ} \mathrm{C}$ for $1 \mathrm{~h}$. The seed solution used for high-density seed deposition was $20 \mathrm{ml}$ of an ethanol solution containing $0.05 \mathrm{~g}$ $\mathrm{ZnAc}_{2} \cdot 2 \mathrm{H}_{2} \mathrm{O}$. After repeated spin coating (2-5 times), the substrates were heated at $300^{\circ} \mathrm{C}$ for $1 \mathrm{~h}$. Coatings with parallel scale-like nanostructures were grown on low-seed-density substrates from a solution containing $1 \mathrm{~g} \mathrm{H}_{2} \mathrm{O}$ and $12 \mathrm{ml}$ EG at $130^{\circ} \mathrm{C}$ for $5 \mathrm{~h}$, whereas coatings with parallel scale-like nanostructures mixed with some tilted scales were obtained with the low-density seed-precoated substrates from a reaction solution containing $0.42 \mathrm{~g} \mathrm{H}_{2} \mathrm{O}$ and $6 \mathrm{ml} \mathrm{EG}$ at $150{ }^{\circ} \mathrm{C}$ for $5 \mathrm{~h}$. The coatings with tilted scale-like nanostructures were grown on high seed density substrates from the solution containing $0.42 \mathrm{~g} \mathrm{H}_{2} \mathrm{O}$ and $12 \mathrm{ml} \mathrm{EG}$ at $150^{\circ} \mathrm{C}$ for $5 \mathrm{~h}$. In some cases, a shorter reaction time proved helpful to grow the nanostructures with lower growth stress.

\section{Surface modification}

Surface modification via perfluorinated silane molecules $(1 \mathrm{H}, 1 \mathrm{H}$, $2 \mathrm{H}, 2 \mathrm{H}$-perfluorooctyltriethoxysilane, PFOTES) was performed with a room-temperature vacuum deposition approach. In detail, one drop of PFOTES liquid was placed beside the bio-inspired coatings; then, low vacuum $(\sim 250 \mathrm{~Pa})$ was applied to the system. After allowing deposition for $24 \mathrm{~h}$, a thin layer of PFOTES molecules deposited on the surface bio-inspired nanostructured coatings.

\section{Characterization}

The preferred orientations of the as-prepared bio-inspired nanostructured coatings were evaluated using a powder X-ray diffractometer (MMA, GBC Scientific Equipment LLC, Hampshire, IL, USA) operating under $\mathrm{Cu} \mathrm{K \alpha}$ radiation. The morphology of the samples was observed with a scanning electron microscope (JSM-7500FA, JEOL, Tokyo, Japan). High-resolution transmission electron microscope observations were carried out using a JEM-2011 F instrument (JEOL) operated at $200 \mathrm{kV}$. Nanoindentation testing was carried out with an Ultra-Micro Indentation System (UMIS-2000, CSIRO, Sydney, NSW, Australia), and a Berkvich tip with a radius of $200 \mathrm{~nm}$ was used to test the hardness and to determine Young's modulus. Incremental loading and unloading tests were performed under load control, and the maximum load was 
set at $1 \mathrm{mN}$. For each of the loading test segments, the loading rate from the initial to the final load occurred in 200 incremental loading steps. The unloading rate from the initial unload to the final unload occurred in 200 decremental unloading steps. Variable-angle reflectance spectra were collected on the thin films comprising $\mathrm{ZnO}$ bio-inspired nanostructures by using an ultravioletvisible-near-infrared light absorption spectroscope operating over the wavelength range of 300-800 nm, and the incident angles of light ranged from 20 to $70^{\circ}$. The spectroscope was equipped with a variable-angle specular reflectance accessory (Cary 500, Agilent, Santa Clara, CA, USA). Wettability and water droplet sliding tests of the bio-inspired nanostructured coatings were conducted by dropping $4-\mu \mathrm{l}$ water droplets on the surface and examining the contact angle and sliding via a Dataphysics OCA20 CA system (Filderstadt, Germany).

\section{RESULTS AND DISCUSSION}

\section{Synthesis of the bio-inspired nanostructures}

Figures $1 \mathrm{c}$ and $\mathrm{d}$ show the microstructure of the fish-scale bio-inspired isolated $\mathrm{ZnO}$ microspheres that were synthesized at $110^{\circ} \mathrm{C}$ for $15 \mathrm{~h}$ from the 4-day-aged reaction solution. The obtained bio-inspired microspheres were $\sim 2 \mu \mathrm{m}$ in diameter, and the surfaces were fully covered with fish-scale-like nanostructures, which exhibited a similar stacking sequence to that of natural fish scales, except that the size of the bio-inspired scales was in the range $200-500 \mathrm{~nm}$; that is, much smaller than real fish scales. The synthesis of these highly ordered stacked fish-scale bio-inspired microspheres was unfortunately very sensitive to preparation parameters, such as the amount of precursors and surfactants added and, particularly, the aging time. Figure 1e presents high-resolution transmission electron microscopy image of one fish-scale-like nanostructure. The figure shows that the exposed basal planes of the scales are precisely the (001) planes of wurtzite $\mathrm{ZnO}$. These well-sequenced, fish-scale-like, nanostructure-covered microspheres would be of great interest for their potential in applications involving micro-/nano-fluidity.

Compared with using isolated bio-inspired microspheres, it would be much more attractive if we could prepare fish-scale-like nanostructures on certain substrates as coatings to obtain low-friction/ low-drag surfaces, anti-corrosion coatings, superhydrophobic or superhydrophilic surfaces and so on. Fish swiftly responds to the external stimulations by changing the orientation of their scales. To study the effect of fish-scale orientation, the bio-inspired coatings with fish-scale-like nanostructures were synthesized with different scale orientations on glass substrates, mainly by controlling the seed density on the substrates and the synthesis temperature, as shown in Figure 2. The substrates used for optical coatings must be extremely clean and smooth. Figure $2 \mathrm{a}$ shows the growth of the bio-inspired coating with parallel scales on a glass substrate that was precoated with a layer of low-density seeds that were crystallized at $400^{\circ} \mathrm{C}$. The fish-scale-like nanostructures on the coating have exposed basal planes; $\mathrm{ZnO}$ (002) planes, parallel to the substrate, were obtained on a substrate that had been precoated at a low seed density at $130^{\circ} \mathrm{C}$ for $5 \mathrm{~h}$. When the synthesis temperature was increased to $150^{\circ} \mathrm{C}$, a fish-scale bio-inspired nanostructured coating with (002) preferred orientation, but mixed with some tilted scales, was obtained on a substrate that had been precoated with low-density seeds (Figure $2 \mathrm{~b}$ ). However, the scales on a bio-inspired coating that had been grown on a glass substrate that was precoated with a layer of high-density seeds that had been crystallized at $300^{\circ} \mathrm{C}$ were tilted with respect to the substrates at angles of $\sim 60-80^{\circ}$ (Figure $2 \mathrm{c}$ ) when synthesized at $150^{\circ} \mathrm{C}$ for $5 \mathrm{~h}$. Figure $2 \mathrm{~d}$ illustrates the growth of the scale-like nanostructures on the substrates under different synthesis conditions. The X-ray diffraction patterns shown in Supplementary Figure S1 demonstrate the preferred orientations of the fish-scale bio-inspired nanostructured coatings: the coating with parallel scales presents the strongest diffraction of
$\mathrm{ZnO}$ (002) planes, whereas the coating with tilted scales shows a significant $\mathrm{ZnO}$ (101) orientation. When the precoated $\mathrm{ZnO}$ seed density was too low, isolated half-spherical islands with highly oriented fish-scale nanostructures were obtained on the glass substrates, as shown in Supplementary Figure S2. The thickness of the fish-scale bioinspired coatings was determined from scanning electron microscope cross-sectional views. As shown in the representative image presented in Supplementary Figure S3, the bio-inspired coatings comprised a top scale-like nanostructured layer and an inner mesoporous interconnected layer, with a total thickness of $\sim 1.2 \mu \mathrm{m}$. The amount of added precursors influenced the size of the fish-scale-like nanostructures, but did not appear to affect the morphology greatly. The hydrothermal synthesis of $\mathrm{ZnO}$ nanostructures can be completed in $30 \mathrm{~min}^{21-22}$ In this study, to ensure the homogenous growth of the thin film and to decrease the possible amorphous layer on the surface, the hydrothermal processes were continued for up to $15 \mathrm{~h}$.

\section{Optical properties of the bio-inspired nanostructures}

Camouflage protection is an important function of natural fish scales and enables fish to escape from predators via light reflection and refraction that can hide the fish or allow them to blend into their environment. ${ }^{10}$ The optical properties of the fish-scale bio-inspired nanostructured coatings with different scale orientations were examined via a variable-angle specular reflectance system that was installed in an ultraviolet-visible-near-infrared spectrometer.

Figure 3 presents reflection spectra and corresponding optical images of the bio-inspired thin films, which show obvious color changes with different angles of tilt to the light source. To exclude the influence of the glass substrates, reflection spectra were also collected on bare substrates, as presented in Supplementary Figure S4. The reflectance of the glass substrates in the measured wavelength range and light-incident angles were $<2.5 \%$, and no influence on the optical properties of the bio-inspired coatings was observed. The reflectance spectra of the bio-inspired coatings were measured from 20 to $70^{\circ}$ at intervals of $10^{\circ}$ by changing the incident light angles. Figure 3 a shows the reflectance spectra of the coating with parallel scale-like nanostructures. Although the reflectance in the yellow-red region was slightly stronger, especially at lower angles of incidence, the overall reflections were significantly enhanced from $\sim 30$ to $\sim 70 \%$ as the angle of the incident light increased, indicating that the bio-inspired coating with parallel scales works similarly to a series of mirror arrays. The inset optical image shows that the bio-inspired coating predominantly reflected yellow colors under white incident light. Figure $3 \mathrm{~b}$ presents the reflections from the bio-inspired coating with a small amount of tilted scale structures. Obvious color variation was observed under white incident light, owing to the refraction caused by the tilted scale-like nanostructures, which work as prism arrays to refract and disperse light. The reflection spectra also revealed that the color of the coatings varied with the incident angle. On the basis of reflection spectra, the coating should appear mainly green under incident light at $20^{\circ}$ and $40-50^{\circ}$, and is expected to appear orange at $30^{\circ}$ and $60^{\circ}$. The bio-inspired coatings with tilted scales (Figure 3c) presented much stronger refraction and color variation, but exhibited much weaker reflections compared with the coatings with parallel scales. The bio-inspired coatings with tilted scales showed predominantly green colors at $20^{\circ}$ and $30^{\circ}$ but predominantly red colors at $50^{\circ}$. Unlike the bio-inspired coatings with parallel scales, in which yellow colors were dominant under white light, the coating with tilted scales presented clear rainbow-like strips. The results indicate that the flat exposed basal planes of the parallel scales function as mirror arrays, whereas the tilted scales act more like prism arrays. 


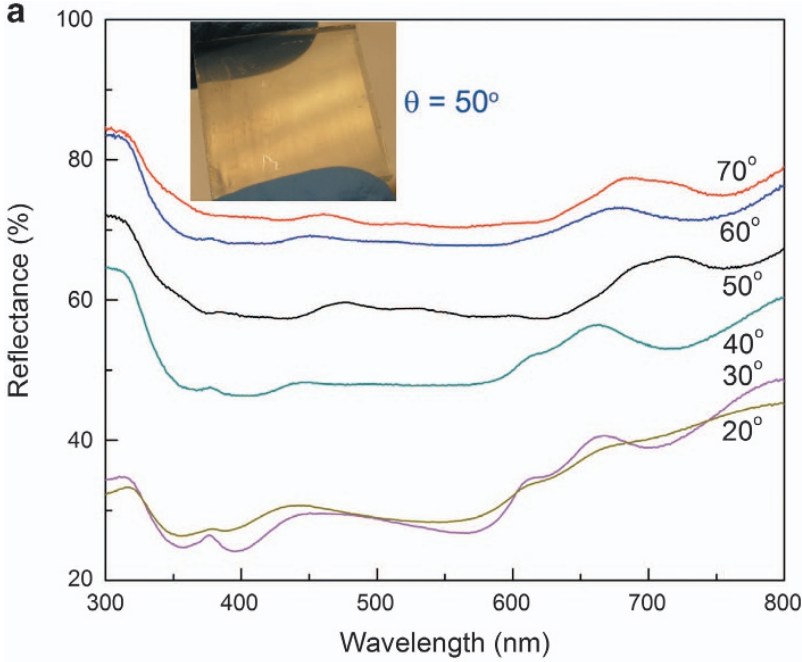

b
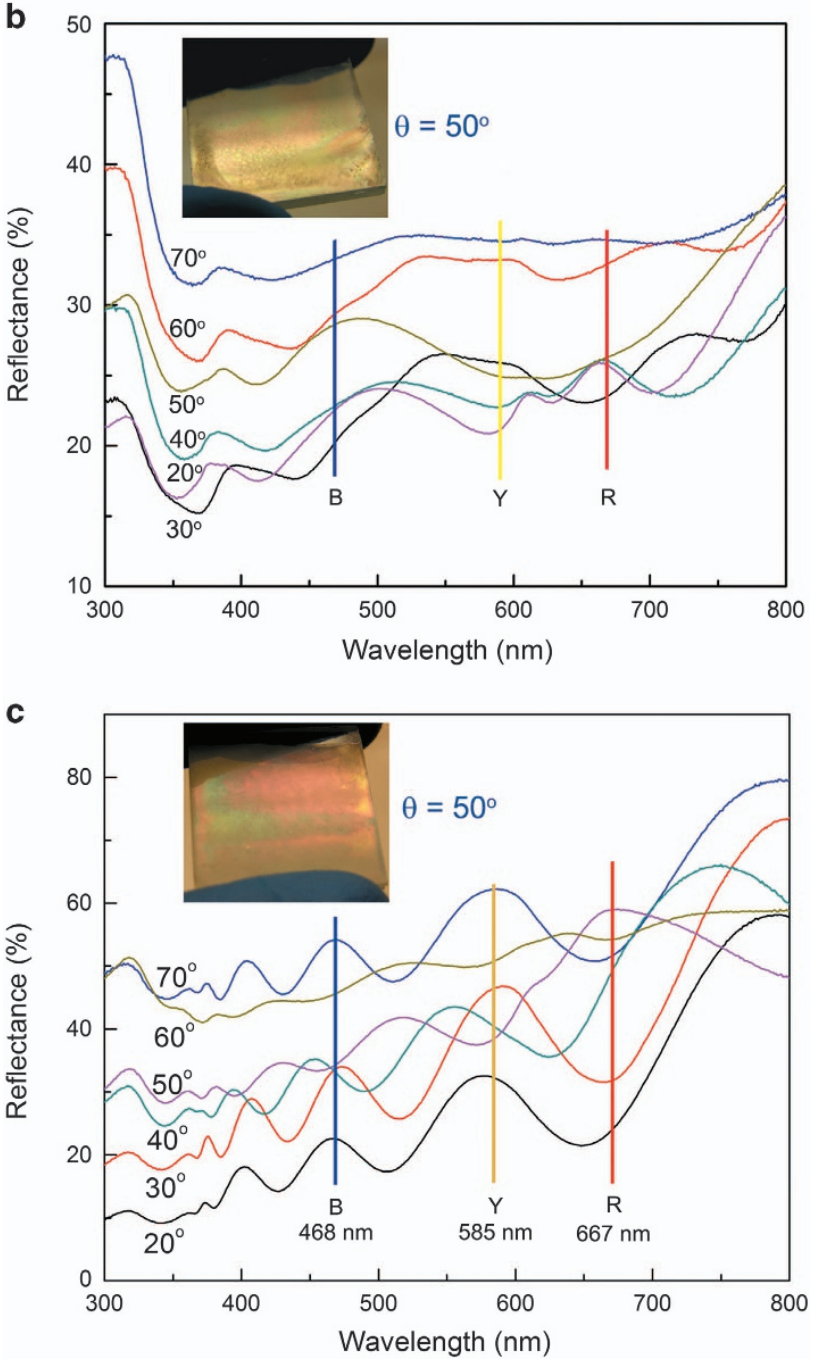

Figure 3 Optical properties of fish-scale bio-inspired nanostructured coatings. Variable-angle reflectance spectra of the bio-inspired coatings with (a) parallel scales, (b) parallel scales mixed with slightly tilting scales and (c) tilting scales. The insets are the corresponding optical images of the bioinspired nanostructured coatings with an incident angle of $\sim 50^{\circ}$ under white light.
The formation of colorful strips on the bio-inspired coatings might result from domains where the tilted scale-like crystals face in different directions. The rainbow-like, shimmering iridescence that resulted from the specific hierarchically ordered nanostructures in the fish-scale bio-inspired nanostructures is not only of great scientific interest but also might prove useful in a wide range of applications related to reflective displays, packaging, advertising and solar energy harvesting. ${ }^{23-26}$ The fish-scale bio-inspired nanostructured coatings provide a new way to design thin films or coatings with tunable optical properties for use in modern optoelectronic devices.

\section{Surface properties of the bio-inspired nanostructures}

As an interface between the inner organs and the water environment, fish scales have a key role in protecting fish from contamination by oil pollution and exhibit self-cleaning and anti-fouling properties. ${ }^{10}$ It was reported that natural fish scales provide underwater superoleophobicity. ${ }^{13}$ In this study, we found that the fish-scale bio-inspired nanostructured coatings with tailored scale orientations exhibited tunable wettability in response to surface modification. Figure 4a presents our concept for tuning the surface wettability of the fish-scale bio-inspired nanostructures by depositing perfluorinated silane (PFOTES) molecules or P123 molecules, respectively. When we applied perfluorinated silane to the $\mathrm{ZnO}$ surface, the silane head group formed a covalent bond by dehydration between the $-\mathrm{CH}_{2} \mathrm{CH}_{3}$ group in the head of the silane and an -OH group on the surface of the $\mathrm{ZnO}$; in addition, the perfluorinated backbone provides a low surface energy that resists adsorption of water and enhances the hydrophobicity of the surface. ${ }^{27}$ Conversely, when P123 tails were exposed, hydrocarbon groups that are very reactive to water adsorbed free water molecules, resulting in a superhydrophilic surface.

Figures $4 \mathrm{~b}-\mathrm{d}$ presents the wettability of the bio-inspired coatings with different scale orientations before and after surface modification. As shown in Figure 4b, the pristine bio-inspired coating with parallel scales was hydrophobic with a contact angle of $89^{\circ}$. After modifying the coating with $\mathrm{P} 123$, the coating wettability was altered from hydrophobic to superhydrophilic with a contact angle of almost $0^{\circ}$. When a few layers of PFOTES molecules were deposited, the bio-inspired nanostructured coating exhibited hydrophobicity with a contact angle of $118^{\circ}$. The pristine surface of the coating with slightly tilting scales had a contact angle of $110^{\circ}$ when tested for wettability. The silane-treated surface almost became superhydrophobic, showing a contact angle of $142^{\circ}$, whereas the P123-coated surface became superhydrophilic (Figure 4c). The bio-inspired coatings with tilted scales presented much more interesting behavior in response to water. As shown in Figure 4d, the contact angle of the pristine coating was $\sim 125^{\circ}$. The angle was almost $0^{\circ}$ for the P123-decorated surface, and the surface presented excellent superhydrophobicity with a contact angle of $162^{\circ}$ after a PFOTES layer was deposited. The deviations of the measured contact angles were $<3^{\circ}$. Interestingly, the contact angle of the pristine bio-inspired coatings increased with the degree of tilting of the scale-like nanostructures. This increase in contact angle is attributed to the increased surface roughness. Micro-grooves between the tilted scales can trap air inside, resulting in the liquid droplet adopting a Cassie-Baxter state. ${ }^{28}$

To determine the superhydrophobic properties of the materials, it is necessary to determine not only the apparent water contact angle but also the dynamic contact angle (the sliding angle or tilting angle at which the water droplet rolls off an inclined surface). Figure $4 \mathrm{e}-\mathrm{g}$ presents the sliding behavior of water droplets from PFOTES-modified bio-inspired nanostructured coatings when their substrates were tilted at $3-4^{\circ}$. The bio-inspired coatings with parallel scales showed a high 

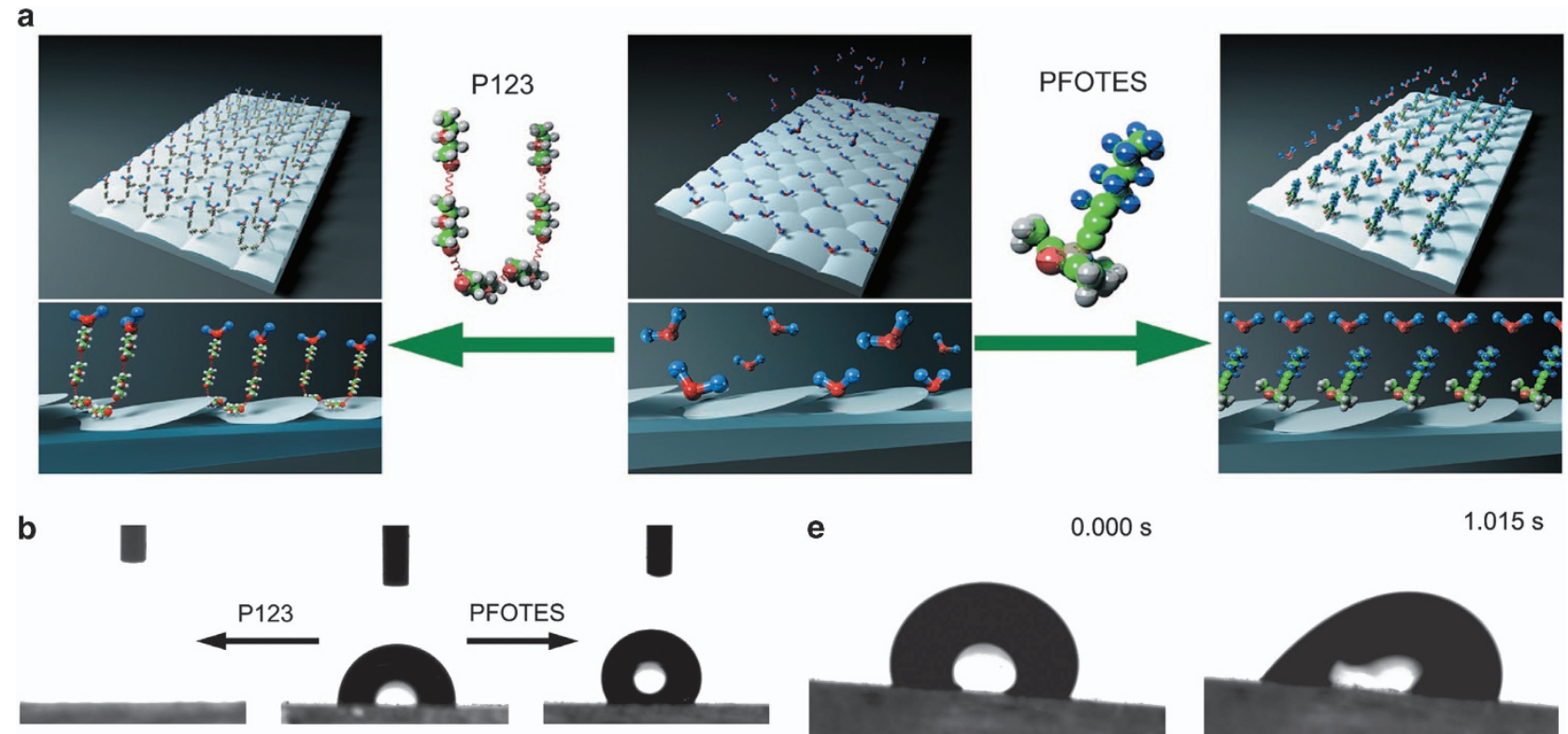

e

$0.000 \mathrm{~s}$

$1.015 \mathrm{~s}$
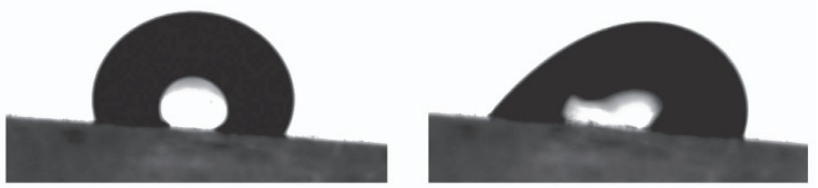

C

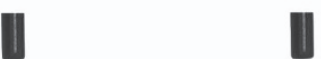

f

$0.000 \mathrm{~s}$

$0.940 \mathrm{~s}$
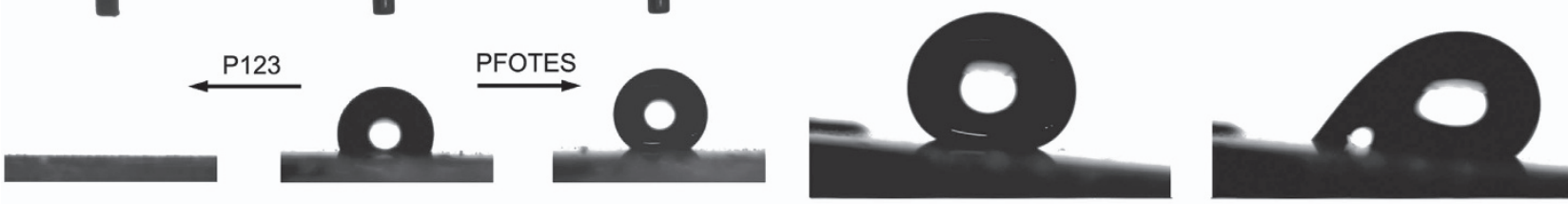

d
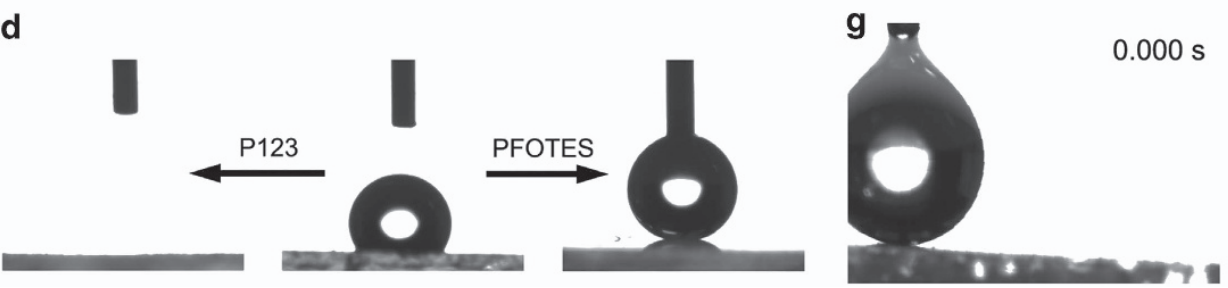

$-$

$0.042 \mathrm{~s}$

Figure 4 Surface wettability modulation of the fish-scale-inspired nanostructured coatings. (a) Schematic drawing of the surface wettability modulation of the fish-scale bio-inspired nanostructures after depositing perflourinated silane $(1 \mathrm{H}, 1 \mathrm{H}, 2 \mathrm{H}, 2 \mathrm{H}$-perfluorooctyltriethoxysilane, PFOTES) and P123 molecules; optical images of the static contact angle of $4-\mu l$ water droplets on the bio-inspired nanostructure coatings with (b) parallel scales, (c) parallel scales mixed with slightly tilted scales and (d) tilted scales, showing the hydrophobic-superhydrophilic-superhydrophobic modulation of the water response; (e) the sliding off after 3-4 $4^{\circ}$ tilting of a $4-\mu \mathrm{l}$ water droplet on bio-inspired nanostructure coatings with (e) parallel scales, (f) parallel scales mixed with slightly tilted scales and $(\mathrm{g})$ tilted scales.

degree of adhesion, as shown in Figure 4e, and water droplets adhered to the tilted bio-inspired coating surface and did not slip off. This suggests that the water droplet on the bio-inspired coating with parallel, flat scale nanostructures almost obeys the Young-Dupre equation. $^{29}$ Even though the bio-inspired coatings with mixing parallel-tilted scales appeared superhydrophobic to the water droplet, high adhesion was observed, as shown in Figure 4f, which shows that the water droplet adhered onto the tilted bio-inspired coating surface and did not slip off, indicating that the surface roughness was insufficient to reach a Cassie-Baxter state. The PFOTES-modified surface of the bio-inspired coating with tilted scales presented not only a high contact angle but also low energy toward a moving water droplet, or a low tilting angle (Figure $4 \mathrm{~g}$ ). It is surprising that water droplets rolled off within $0.1 \mathrm{~s}$ from the planes with a tilting angle of $3^{\circ}$. The high water contact angle and the low sliding angle of the bio-inspired coating with tilted scales indicate that the water droplets follow the Cassie-Baxter equation and do not penetrate into the grooves. ${ }^{30-31}$ High contact angles and low sliding angles are responsible for the self-cleaning properties observed in some natural species, such as cicada wings, and lotus and peanut leaves. $^{32-34}$

On the basis of the static wettability and sliding-off tests, it was concluded that both the wettability and the adhesion behavior of the fish-scale bio-inspired coatings can be tailored by changing the orientation of the scales or by modulating the surface functional groups. In particular, the PFOTES-modified bio-inspired coatings with tilted scales exhibited low surface energy and suitable surface roughness that prevented the adhesion of even tiny water droplets, thus demonstrating that the fish-scale bio-inspired nanostructures are promising superhydrophilic/superhydrophobic materials that might be useful for their anti-icing, anti-fogging, anti-corrosion, anti-bacterial and self-cleaning properties. ${ }^{35}$ 


\section{Mechanical properties of the bio-inspired nanostructures}

One of the most important functions of natural fish scales is to provide sufficient mechanical protection for the inner soft organs. The fish-scale bio-inspired inorganic nanostructures are also expected to provide significant mechanical tolerance that meets the requirements for multifunctional applications. The mechanical properties of the fish-scale bio-inspired nanostructured coatings were examined via nanoindentation testing. Figure 5 shows the nanoindentation behavior of the fish-scale bio-inspired nanostructured coatings with different preferred scale orientations. Figures $5 \mathrm{a}-\mathrm{c}$ display typical load-displacement curves of the fish-scale bio-inspired nanostructured coatings with parallel scales (coating with the (002) preferred orientation), with slightly tilted scales (coating with the (002) preferred orientation but mixing with small portion of tilted nanostructures) and with tilted scales (coating with the (101) preferred orientation), respectively. The loading-unloading curves obtained for the bio-inspired coatings are also presented in Supplementary Figure S5. Clearly, the differences in the preferred orientation of the scale-like nanostructures resulted in entirely different mechanical responses of the bio-inspired coatings. The bio-inspired coatings with parallel and flat scales (Figure 5a and Supplementary Figure S5(a)) and the coatings with slightly tilted scales (Figure $5 \mathrm{~b}$ and Supplementary Figure S5(b)) allowed much deeper displacement compared with the $200 \mathrm{~nm}$ maximum that was obtained for the coating with tilted scales (Figure 5c and Supplementary Figure S5(c)), and the clear residual depths observed after unloading confirmed the plastic nature of both nanostructures.

The variations of the hardness $(H)$ and elastic modulus $(E)$ of the bio-inspired coatings as functions of the maximum displacement are plotted in Figures 5d-f. By analyzing the variations in the hardness and the elastic modulus of the coatings with parallel scales and slightly tilted scales, it was found that the values of $H$ and $E$ both decreased exponentially and reached a plateau when the displacement was $>\sim 500 \mathrm{~nm}$, indicating that the indenter had reached the softer inner layers. The large loading-unloading loops in Figures $5 \mathrm{a}$ and $\mathrm{b}$ and Supplementary Figure S5(a-b) indicate low plastic deformation resistance along the direction perpendicular to the exposed planes (the [001] direction). In other words, the parallel scales with plentiful a
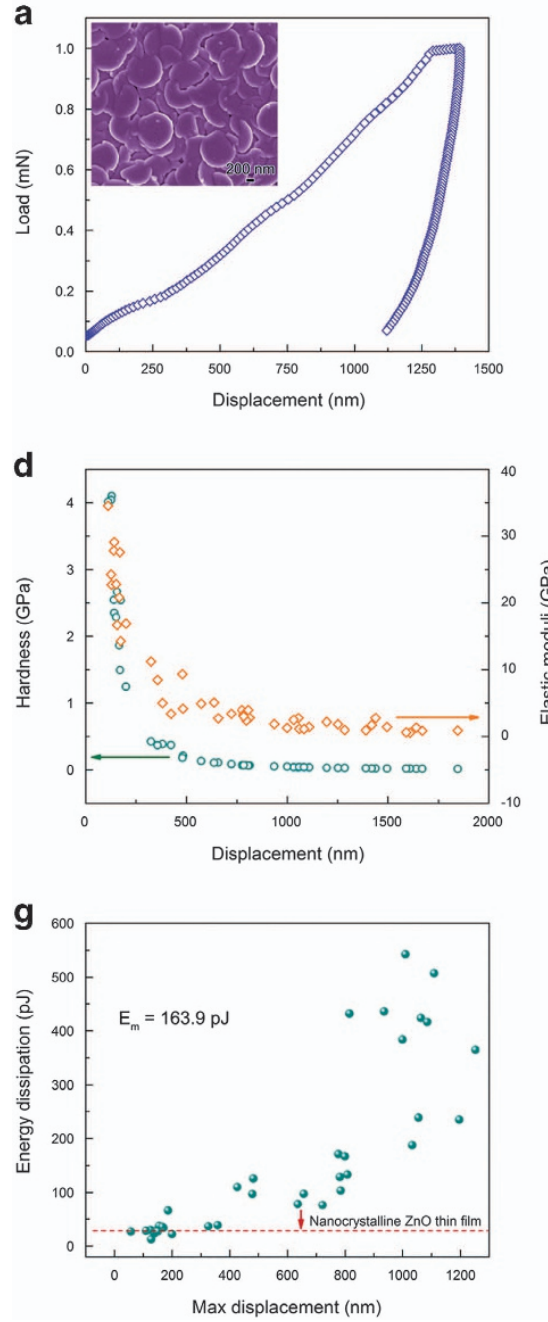

b
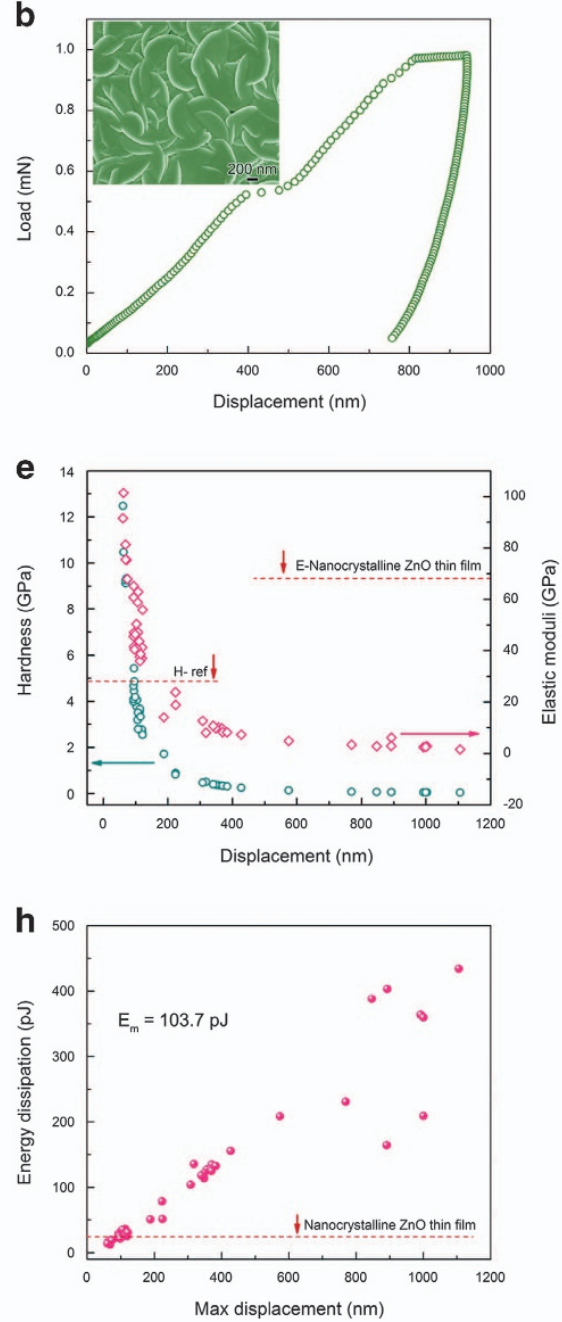
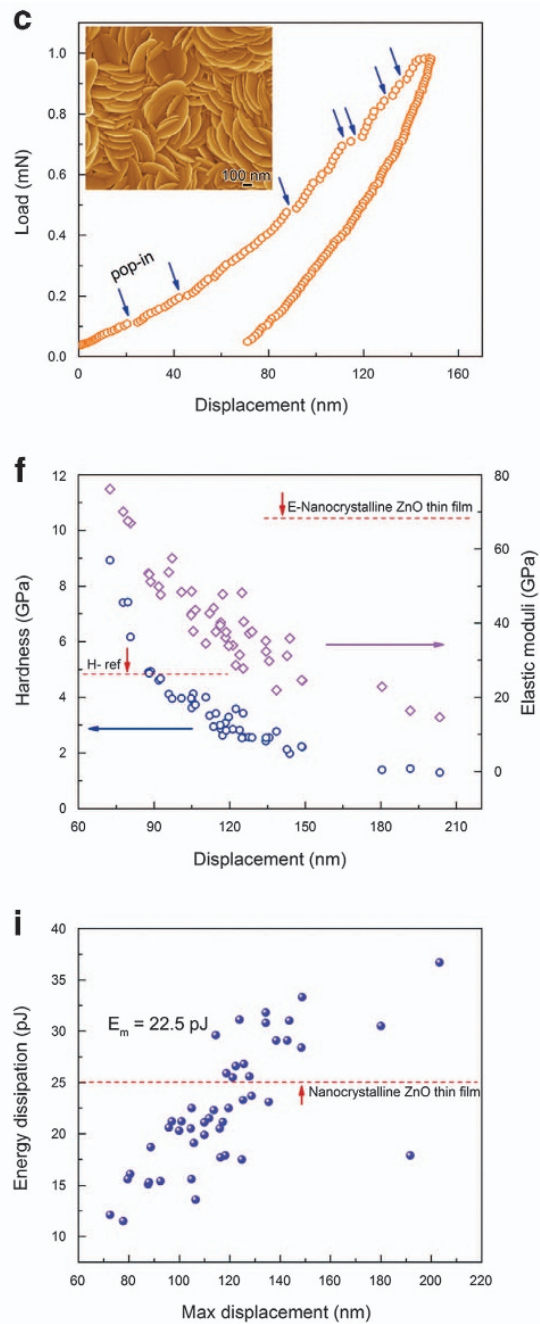

Figure 5 Mechanical properties of fish-scale bio-inspired nanostructure coatings. Typical nanoindentation load-displacement curves for bio-inspired nanostructure coatings with (a) parallel scales, (b) parallel scales mixed with slightly tilting scales and (c) tilting scales; variation of the hardness and elastic modulus as functions of indentation depth of the bio-inspired coatings with (d) parallel scales, (e) parallel scales mixed with slightly tilted scales and (f) tilted scales; and variation of energy dissipation as a function of maximum indentation depth of the bio-inspired coatings with (e) parallel scales, (h) parallel scales mixed with slightly tilted scales and (i) tilted scales. The dashed lines in (d-i) indicate the mechanical properties of the reference ZnO nanocrystalline thin film. 
thin edges are softer and more favorable for plastic deformation during the indentation process, which results in low values of $H\left(H_{\text {mean }}=0.70 \pm 1.17 \mathrm{GPa}\right)$ and $E\left(E_{\text {mean }}=7.44 \pm 9.36 \mathrm{GPa}\right)$ of the parallel scale coatings (Figure 5d) and slightly higher values of $H\left(H_{\text {mean }}=2.83 \pm 3.21 \mathrm{GPa}\right)$ and $E\left(E_{\text {mean }}=32.28 \pm 27.54 \mathrm{GPa}\right)$ for the coatings with slightly tilting scales (Figure 5e). When the indentation direction was mostly perpendicular to the $\{101\}$ planes (as shown in Figure 5f), the tilted scales exhibited much higher values of hardness $\left(H_{\text {mean }}=3.55 \pm 1.62 \mathrm{GPa}\right)$ and elastic modulus $\left(E_{\text {mean }}=40.86 \pm 13.50 \mathrm{GPa}\right)$. Moreover, the values obtained on the tilted coatings did not sharply decrease with increasing displacement, and the scales were much more resistant to mechanical deformation. The higher hardness of the bio-inspired coatings with tilted scales can be attributed to their stacking mode; in this mode, the scales are packed against each other very closely, and insufficient space is present to allow deformation and cracking of the scale-like nanostructures. The measured hardness and elastic modulus of the bio-inspired coatings with tilted scales coincide well with reported data on nanocrystalline $\mathrm{ZnO}$ thin films, which are indicated by the dashed lines in Figure $5{ }^{36-37}$ It has been reported that bulk $\mathrm{ZnO}$ material with basal planes lying perpendicular to the surface is more susceptible to slip and might be reflected in the occurrence of large pop-in events for indentations. ${ }^{38-39}$ Many pop-in events can be found in the curves describing the indentation of the bio-inspired thin films with tilted scales (Figure $5 \mathrm{c}$ ). The tilted scales were much harder and tougher than the parallel scales, indicating that the mechanical properties of the hierarchically ordered nanostructures depend not only on the crystal structure of the material but also on the stacking mode.

On the basis of the nanoindendation results, it is concluded that the fish-scale bio-inspired nanostructures, especially those with parallel scales, possess the capability to dissipate the energy of mechanical damage and present damage-tolerant features; thus, the nanostructures can protect the inner weak substrates from catastrophic failure. The damage tolerance of the coatings can be further confirmed by the energy dissipated during mechanical deformation (Figures $5 \mathrm{~g}-\mathrm{i}$ ), that is, the area enclosed by the loading-unloading curves. The amount of mechanical energy dissipation can provide a quantitative evaluation of the damage energy absorbed during mechanical damage and can indicate the capacity of the materials to prevent catastrophic failure resulting from mechanical impact. ${ }^{11,40}$ The bio-inspired coatings with parallel scales presented much larger mechanical loops than the reference $\mathrm{ZnO}$ nanocrystalline thin film and the bio-inspired coatings with tilted scales. As shown in Figures $5 \mathrm{~g}-\mathrm{i}$, the bio-inspired coating with parallel scales and the coating with slightly tilted scales exhibited significant energy dissipation, on the order of hundreds of pJ, and reached average values of $163.86 \pm 161.99$ and $103.67 \pm 151.09 \mathrm{pJ}$, respectively. Nevertheless, the mean energy dissipation for the bioinspired coatings with tilted scales was only $22.5 \pm 6.1 \mathrm{pJ}$. The values obtained for the coating with tilted scales are quite close to the energy dissipation of the reference $\mathrm{ZnO}$ nanocrystalline thin films that lacked a preferred orientation, as shown by the dashed lines in the figures $(\sim 25 \mathrm{pJ}) .{ }^{36-37}$ Obviously, the mechanical properties of the fish-scale bio-inspired nanostructures are strongly correlated with the scale-stacking mode, and bio-inspired nanostructured coatings with tailored mechanical properties can be designed to meet the requirements of various application environments.

\section{CONCLUSION}

In summary, inspired by multifunctional fish scales with swift stimulus-responsive abilities, we developed inorganic nanostructured isolated particles and nanostructured coatings with surface morphologies that mimic those of natural fish scales via a facile self-assembly approach. The fish-scale bio-inspired nanostructured coatings feature tunable light refraction and reflection, tunable surface wettability and damage-tolerant mechanical properties. The salient properties of the fish-scale bio-inspired multifunctional nanostructures hold promise for use in the following applications: (i) optical coatings, sensing or lens arrays in reflective displays, packaging, advertising and solar energy harvesting; (ii) self-cleaning surfaces, including anti-smudge, anti-fouling and anti-fogging, and self-sterilizing surfaces; and (iii) mechanically/chemically protective coatings. Moreover, the facile self-assembly method allows the low-cost and large-scale fabrication of these bio-inspired nanostructures. Therefore, we believe that the fish-scale-inspired nanostructured microspheres and coatings represent a new platform for developing novel functional materials for use in 'smart' structures and other applications.

\section{CONFLICT OF INTEREST}

The authors declare no conflict of interest.

\section{ACKNOWLEDGEMENTS}

This work was partly supported by the Australian Research Council Discovery Project (DP1096546), a Discovery Early Career Researcher Award (DE150100280), the National Natural Science Foundation of China (21273016 and 21001013), the National Basic Research Program of China (2013CB933003), the Program for New Century Excellent Talents in Universities, Beijing Natural Science Foundation (2122035). T.L. was also supported by a UOW Vice-Chancellor's (VC) Research Fellowship. W.X.L. acknowledges research support by the Program for Professor of Special Appointment (Eastern Scholar) at Shanghai Institutions of Higher Learning.

1 Jiang, L. \& Feng, L. Bioinspired Intelligent Nanostructured Interfacial Materials (World Scientific Publishing Co. Pty Ltd and Chemical Industry Press, Singapore, 2010).

2 Kumar, C. Biomimetic and Bioinspired Nanomaterials (Wiley-VCH Verlag Gmbh \& Co., Weinheim, Germany, 2010).

3 Li, K., Xue, Z., Ma, J., Feng, L., Gao, S. \& Jiang, L. Structured cone arrays for continuous and effective collection of micron-sized oil droplets from water. Nat. Commun. 4, 2276 (2013).

4 Yang, S. Y., O'Cearbhaill, E. D., Sisk, G. C., Park, K. M., Cho, W. K., Villiger, M., Bouma, B. E., Pomahac, B. \& Karp, J. M. A bio-inspired swellable microneedle adhesive for mechanical interlocking with tissue. Nat. Commun. 4, 1702 (2013).

5 Galusha, J. W., Jorgensen, M. R. \& Bartl, M. H. Diamond-structured titania photonicbandgap crystals from biological templates. Adv. Mater. 22, 107-110 (2010).

6 Saranathan, V., Osuji, C. O., Mochrie, S. G., Noh, H., Narayanan, S., Sandy, A. Dufresne, E. R. \& Prum, R. O. Structure, function, and self-assembly of single network gyroid (14132) photonic crystals in butterfly wing scales. Proc. Natl Acad. Sci. USA 107, 11676-11681 (2010).

7 Sun, Z., Liao, T., Liu, K., Jiang, L., Kim, J. H. \& Dou, S. X. Fly-eye inspired superhydrophobic anti-fogging inorganic nanostructures. Small 10 3001-3006 (2014).

8 Yao, H., Dao, M., Imholt, T., Huang, J., Wheeler, K., Bonilla, A., Suresh, S. \& Ortiz, C. Protection mechanisms of the iron-plated armor of a deep-sea hydrothermal vent gastropod. Proc. Natl Acad. Sci. USA 107, 987-992 (2010).

9 Jin, X., Shi, B., Zheng, L., Pei, X., Zhang, X., Sun, Z., Du, Y., Kim, J. H., Wang, X., Dou, S., Liu, K. \& Jiang, L. Bio-inspired multifunctional metallic foams through the fusion of different biological solutions. Adv. Funct. Mater. 24, 2721-2726 (2014).

10 Helfman, G. S., Collette, B. B., Facey, D. E. \& Bowen, B. W. The Diversity of Fishes: Biology, Evolution, and Ecology. 2nd ed.(Wiley-Blackwell, MA, USA, 2009).

11 Bruet, B. J. F., Song, J., Boyce, M. C. \& Ortiz, C. Materials design principles of ancient fish armour. Nat. Mater. 7, 748-756 (2008).

12 Oeffner, J. \& Lauder, G. V. The hydrodynamic function of shark skin and two biomimetic applications. J. Exp. Biol. 215, 785-795 (2012).

13 Liu, M., Wang, S., Wei, Z., Song, Y. \& Jiang, L. Bioinspired design of a superoleophobic and low adhesive water/solid interface. Adv. Mater. 21, 665-669 (2009).

14 Kumazawa, Y. \& Nishida, M. Molecular phylogeny of osteoglossoids: a new model for Gondwanian origin and plate tectonic transportation of the Asian arowana. Mol. Biol. Evol. 17, 1869-1878 (2000).

15 Djurisic, A. B., Chen, X., Leung, Y. H. \& Ng, A. M. C. ZnO nanostructures: growth, properties and applications. J. Mater. Chem. 22, 6526-6535 (2012). 
16 Sun, Z., Liao, T., Dou, Y., Hwang, S. M., Park, M. S., Jiang, L., Kim, J. H. \& Dou, S. X. Generalized self-assembly of scalable two-dimensional transition metal oxide nanosheets. Nat. Commun. 5, 3813 (2014).

17 Sun, Z., Liao, T., Kim, J. G., Liu, K., Jiang, L., Kim, J. H. \& Dou, S. X. Architecture designed $\mathrm{ZnO}$ hollow microspheres with wide-range visible-light photoresponses. J. Mater. Chem. C 1, 6924-6929 (2013).

18 Kim, D., Lee, K. Y., Gupta, M. K., Majumder, S. \& Kim, S. W. Self-compensated insulating zno-based piezoelectric nanogenerators. Adv. Funct. Mater. 24, 6949-6955 (2014).

19 Liu, Q., Sun, Z., Dou, Y., Kim, J. H. \& Dou, S. X. Two-step self-assembly of hierarchically-ordered nanostructures. J. Mater. Chem. A 3, 11688-11699 (2015).

20 Wu, Y., Pei, X., Wang, X., Liang, Y., Liu, W. \& Zhou, F. Biomimicking lubrication superior to fish skin using responsive hydrogels. NPG Asia Mater. 6, e136 (2014).

21 Choi, C. H. \& Chang, C. H. Aqueous synthesis of tailored ZnO nanocrystals, nanocrystal assemblies, and nanostructured films by physical means enabled by a continuous flow microreactor. Cryst. Growth Des. 14, 4759-4767 (2014).

22 Han, S. Y., Paul, B. K. \& Chang, C. H. Nanostructured ZnO as biomimetic anti-reflective coatings on textured silicon using a continuous solution process. J. Mater. Chem. 22, 22906-22912 (2012).

23 Chung, K., Yu, S., Heo, C., Shim, J. W., Yang, S. M., Han, M. G., Lee, H., Jin, Y., Lee, S. Y., Park, N. \& Shin, J. H. Flexible, angle-independent, structural color reflectors inspired by morpho butterfly wings. Adv. Mater. 24, 2375-2379 (2012).

24 Diao, Y. Y. \& Liu, X. Y. Controlled colloidal assembly: experimental modeling of general crystallization and biomimicking of structural color. Adv. Funct. Mater. 22, 1354-1375 (2012).

25 Zhu, C., Xu, W., Chen, L., Zhang, W., Xu, H. \& Gu, Z. Magnetochromatic microcapsule arrays for displays. Adv. Funct. Mater. 21, 2043-2048 (2011).

26 Colonna, D., Colodrero, S., Lindstrom, H., Carlo, A. D. \& Miguez, H. Introducing structural colour in DSCs by using photonic crystals: interplay between conversion efficiency and optical properties. Energy Environ. Sci. 5, 8238-8243 (2011).

27 Sun, Z., Liao, T., Liu, K., Jiang, L., Kim, J. H. \& Dou, S. X. Robust superhydrophobicity of hierarchical $\mathrm{ZnO}$ hollow microspheres fabricated by two-step self-assembly. Nano Res. 6, 726-735 (2013).

28 Cassie, A. B. D. \& Baxter, S. Wettability of porous surfaces. Trans. Faraday Soc. 40, 546-551 (1944).

29 Young, T. An Essay on the Cohesion of Fluids. Philos. Trans. R. Soc. London 95, 65-87 (1805)

30 Miwa, M., Nakajima, A., Fujishima, A., Hashimoto, K. \& Watanabe, T. Effects of the surface roughness on sliding angles of water droplets on superhydrophobic surfaces. Langmuir 16, 5754-5760 (2000).
31 Wang, S., Li, Y., Fei, X., Sun, M., Zhang, C., Li, Y., Yang, Q. \& Hong, X. Preparation of a durable superhydrophobic membrane by electrospinning poly (vinylidene fluoride) (PVDF) mixed with epoxy-siloxane modified SiO2 nanoparticles: a possible route to superhydrophobic surfaces with low water sliding angle and high water contact angle. J. Colloid Interface Sci. 359, 380-388 (2011).

32 Yang, S., Ju, J., Qiu, Y., He, Y., Wang, X., Dou, S., Liu, K. \& Jiang, L. Peanut leaf inspired multifunctional surfaces. Small 10, 294-299 (2014).

33 Wisdom, K. M., Watson, J. A., Qu, X., Liu, F., Watson, G. S. \& Chen, C. H. Self-cleaning of superhydrophobic surfaces by self-propelled jumping condensate. Proc. Natl Acad. Sci. USA 110, 7992-7997 (2013).

34 Barthlott, W. \& Neinhuis, C. Purity of the sacred lotus, or escape from contamination in biological surfaces. Planta 202, 1-8 (1997).

35 Darmanin, T. \& Guittard, F. J. Recent advances in the potential applications of bioinspired superhydrophobic materials. Mater. Chem. A 2, 16319-16359 (2014).

36 Mayo, M. J., Siegel, R. W., Liao, Y. X. \& Nix, W. D. Nanoindentation of nanocrystalline ZnO. J. Mater. Res. 7, 973-979 (1992).

37 Fang, T. H., Chang, W. J. \& Lin, C. M. Mater. Sci. Eng. A 452-453, 715-720 (2007).

38 Coleman, V. A., Jagadish, J. in Zinc Oxide Bulk, Thin Films and Nanostructures Vol. 1. (eds Jagadish, C. \& Pearton, S.) (Elsevier, 2006).

39 Coleman, V. A., Bradby, J. E., Jagadish, C., Munroe, P., Heo, Y. W., Pearton, S. J., Norton, D. P., Inoue, M. \& Yano, M. Mechanical properties of $\mathrm{ZnO}$ epitaxial layers grown on a- and c-axis sapphire. Appl. Phys. Lett. 86, 203105 (2005).

40 Mckittrick, J., Chen, P. Y., Tombolato, L., Novitskaya, E. E., Trim, M. W., Hirata, G. A., Olevsky, E. A., Horstmeyer, M. F. \& Meyers, M. A. Energy absorbent natural materials and bioinspired design strategies: A review. Mater. Sci. Eng. C 30, 331-342 (2010).

(i) This work is licensed under a Creative Commons Attribution 4.0 International License. The images or other third party material in this article are included in the article's Creative Commons license, unless indicated otherwise in the credit line; if the material is not included under the Creative Commons license, users will need to obtain permission from the license holder to reproduce the material. To view a copy of this license, visit http://creativecommons.org/licenses/by/4.0/

Supplementary Information accompanies the paper on the NPG Asia Materials website (http://www.nature.com/am) 